\title{
Effects of Ezetimibe-Statin Combination Therapy on Coronary Atherosclerosis in Acute Coronary Syndrome
}

\author{
Kiyoshi Hibi, MD; Shinjo Sonoda, MD; Masanori Kawasaki, MD; Yutaka Otsuji, MD; \\ Toyoaki Murohara, MD; Hideki Ishii, MD; Katsuhiko Sato, MD; Ryoji Koshida, MD; \\ Yukio Ozaki, MD; Masataka Sata, MD; Yoshihiro Morino, MD; Tadashi Miyamoto, MD; \\ Tetsuya Amano, MD; Satoshi Morita, PhD; Ken Kozuma, MD; Kazuo Kimura, MD; \\ Hisayoshi Fujiwara, MD for the Ezetimibe-ACS Investigators
}

\begin{abstract}
Background: The results of previous clinical trials on the effects of ezetimibe-statin combination therapy on atherosclerosis are inconsistent, and the anti-atherosclerotic effect of ezetimibe remains controversial.

Methods and Results: We conducted a prospective, randomized open-label study at 10 centers. One hundred and twenty-eight statin-naïve patients with acute coronary syndrome (ACS) undergoing intravascular ultrasound (IVUS)-guided percutaneous coronary intervention were randomized to receive either $2 \mathrm{mg} /$ day pitavastatin plus $10 \mathrm{mg} /$ day ezetimibe, or $2 \mathrm{mg} /$ day pitavastatin. One hundred and 3 patients had evaluable IVUS of non-culprit coronary lesions at baseline and at follow-up. The primary endpoint was the percentage change in non-culprit coronary plaque volume (PV) and lipid PV on integrated backscatter IVUS. Mean low-density lipoprotein cholesterol was reduced from $123 \mathrm{mg} / \mathrm{dL}$ to $64 \mathrm{mg} / \mathrm{dL}$ in the combination therapy group $(\mathrm{n}=50)$ and $126 \mathrm{mg} / \mathrm{dL}$ to $87 \mathrm{mg} / \mathrm{dL}$ in the statin alone group ( $n=53$; between-group difference, $16.9 \%, P<0.0001)$. The percent change in $P V$ was $-5.1 \%$ in the combination therapy group and $-6.2 \%$ in the statin alone group $(P=0.66)$, although both groups had reduction of $P V$ compared with baseline (both $P<0.01)$. The percent change in lipid $P V$ did not differ between the groups (4.3 vs. $-3.0 \%, P=0.37$ ).
\end{abstract}

Conclusions: In statin-naïve patients with ACS, combined therapy with ezetimibe and statin did not result in a significant change in coronary plaque regression or tissue component compared with statin alone. [Clinical Trial Registration: www.clinicaltrials.gov (NCT00549926)]

Key Words: Acute coronary syndrome; Intravascular ultrasound; Lipid-lowering therapy; Low-density lipoprotein cholesterol

$\bigcirc$ everal randomized clinical trials have shown that lowering low-density lipoprotein cholesterol (LDLC) with high-dose statin reduces LDL-C and improves clinical outcome compared with standard dose statin therapy or placebo. ${ }^{1-3}$ A meta-analysis of 27 such randomized trials concluded that $1.0 \mathrm{mmol} / \mathrm{L}(38.66 \mathrm{mg} / \mathrm{dL})$ reduction in LDL-C by statin resulted in proportional reduction of cardiovascular events by $16 \%$ in women and by $22 \%$ in men. ${ }^{4}$ Double-dose statin, however, offers only limited additional lowering of LDL-C,,5 and high-dose statin therapy is associated with increased incidence of side-effects. ${ }^{7}$

In contrast, several studies have demonstrated that ezetimibe, which reduces cholesterol absorption from the small intestine through inhibition of the Nieman-Pick C1-like1 protein, can produce significant reduction in serum LCL-C and has cardiovascular protective effects. When added to statin, ezetimibe provides an incremental reduction in LDL-C of 16-26\%.5,6 The clinical trial Improved Reduction of Outcomes: Vytorin Efficacy International Trial

Received June 26, 2017; revised manuscript received October 2, 2017; accepted October 13, 2017; released online December 7, 2017 Time for primary review: 4 days

Division of Cardiology, Yokohama City University Medical Center, Yokohama (K.H., K. Kimura); Second Department of Internal Medicine, School of Medicine, University of Occupational and Environmental Health, Kitakyushu (S.S., Y. Otsuji); Department of Cardiology, Gifu University Graduate School of Medicine, Gifu (M.K.); Department of Cardiology, Nagoya University, Nagoya (T. Murohara, H.I.); Tokeidai Memorial Hospital Cardiovascular Center, Sapporo (K.S., R.K.); Department of Cardiology, Fujita Health University School of Medicine, Toyoake (Y. Ozaki); Department of Cardiovascular Medicine, Tokushima University, Tokushima (M.S.); Division of Cardiology, Department of Internal Medicine, Iwate Medical University School of Medicine, Morioka (Y.M.); Department of Cardiovascular Medicine, Hyogo Prefectural Amagasaki Hospital, Amagasaki (T. Miyamoto, H.F.); Department of Cardiology, Aichi Medical University, Nagakute (T.A.); Department of Biomedical Statistics and Bioinformatics, Kyoto University Graduate School of Medicine, Kyoto (S.M.); and Division of Cardiology, Teikyo University School of Medicine, Tokyo (K. Kozuma), Japan

The Guest Editor for this article was Dr. Yoshihiko Saito.

Mailing address: Kiyoshi Hibi, MD, Division of Cardiology, Yokohama City University Medical Center, 4-57 Urafune-cho, Minami-ku, Yokohama 232-0024, Japan. E-mail: hibikiyo@yokohama-cu.ac.jp

ISSN-1346-9843 All rights are reserved to the Japanese Circulation Society. For permissions, please e-mail: cj@j-circ.or.jp 
(IMPROVE-IT) reported the clinical benefits of the combination of ezetimibe and statin during a median follow-up of 6 years. $^{8}$ In a recent randomized study, Tsujita et al demonstrated the superiority of the combination of ezetimibe and statin on coronary atherosclerosis, compared with statin alone, in patients with coronary artery disease. ${ }^{9}$ Similarly, the SANDS clinical trial concluded that the beneficial effects of the combination of ezetimibe plus statin on carotid atherosclerosis was similar to that of statin alone in patients with similar reduction in LDL-C. ${ }^{10}$ Other clinical trials on the combination of ezetimibe and statin on carotid atherosclerosis, however, noted variable results: both beneficial ${ }^{10}$ and non-favorable outcomes. ${ }^{11,12}$ Thus, the anti-atherosclerotic effects of ezetimibe remain controversial. The present prospective, randomized open-label study conducted at 10 centers in Japan, compared the effects of $2 \mathrm{mg} /$ day pitavastatin with those of the combination of $2 \mathrm{mg} /$ day pitavastatin plus $10 \mathrm{mg}$ /day ezetimibe on percent change in coronary plaque volume (PV) and lipid PV, as determined on intravascular ultrasonography (IVUS).

\section{Methods}

\section{Subjects}

This prospective, randomized open-label parallel group study with blind endpoint evaluation was conducted at 10 health centers to compare the effects of pitavastatin plus ezetimibe vs. pitavastatin alone on coronary atherosclerosis in statin-naïve patients with acute coronary syndrome (ACS). All subjects were diagnosed with ACS and underwent successful percutaneous coronary intervention (PCI) for the culprit lesion under IVUS guidance. ACS was defined as unstable angina pectoris, non-ST-segment elevation myocardial infarction (NSTEMI) or ST-segment elevation myocardial infarction (STEMI). ${ }^{13} \mathrm{We}$ excluded patients with severely calcified lesions, coronary bypass graft lesion, restenotic lesion, treatment with lipid-lowering agents (statin, niacin, probucol, fibrate, and anion exchange resin), homozygous familial hypercholesterolemia, hemodynamic instability, cardiogenic shock, planned revascularization of the target plaque, history of revascularization of the target plaque, active liver disease (alanine aminotransferase $\geq 100 \mathrm{IU} / \mathrm{L}$ ), or severe renal insufficiency (serum creatinine $\geq 2.0 \mathrm{mg} / \mathrm{dL}$ ). Culprit lesions were classified according to the electrocardiographic change and angiographic appearance during PCI.

A total of 128 patients with ACS who underwent IVUSguided PCI were centrally randomized into the pitavastatin ( $2 \mathrm{mg} /$ day) plus ezetimibe $(10 \mathrm{mg} /$ day) group and pitavastatin monotherapy ( $2 \mathrm{mg} /$ day) group using an internetbased program, and stratified according to hyperlipidemia and diabetes using the minimization method. We used the minimization method to minimize imbalances between important factors in both groups. Changes in lipid profile, $\mathrm{C}$-reactive protein (CRP), and pentraxin 3 were calculated at follow-up and expressed relative to baseline.

The study was registered at www.clinicaltrials.gov (NCT00549926) and conducted according to the Declaration of Helsinki. The study protocol was approved by the institutional human ethics review board of each center and written informed consent was obtained from each patient.

\section{IVUS}

The PCI strategy, such as the decision to stent without balloon pre-dilatation or post-dilatation after stent implan- tation was left to the discretion of the individual operator. After PCI, IVUS was conducted to evaluate non-culprit coronary lesions and this was performed at baseline and at 8-12-month follow-up in 103 patients. After another $200 \mu \mathrm{g}$ nitroglycerin i.c., a 40-MHz IVUS catheter (ViewIT, Terumo, Tokyo, Japan) was advanced over a 0.014-in guidewire and positioned as far distally as could be safely reached, and imaging was performed in a retrograde fashion to the aorto-ostial junction at an automatic pullback speed of $0.5 \mathrm{~mm} / \mathrm{s}$, facilitating the observation of the lesion. IVUS in the non-culprit vessel was strongly recommended, but non-culprit lesion in the culprit vessel was allowed according to the clinical situation in the setting of ACS. IVUS was also performed at 8-12-month follow-up, and the same IVUS imaging system was used in all examinations.

Two independent experienced investigators blinded to the clinical data analyzed the IVUS quantitatively in the independent core laboratory (Cardiocore, Yokohama, Japan). IVUS analysis was performed using a validated planimetry system (Visiatlas ver. 2.0, Terumo). The target segment for analysis was a mild-moderate stenosis in the non-culprit vessel. In the case of non-culprit vessel IVUS deemed impossible by the operators, the non-PCI site of the culprit vessel ( $>5 \mathrm{~mm}$ proximal or distal to the PCI site) was selected. Spotty calcification, side branch, and stent edge were used as reproducible landmarks to synchronize the target plaque at baseline and at follow-up. For each patient, the cross-sectional area (CSA) of the external elastic membrane (EEM) and of the intravascular lumen was measured according to the standards of the American College of Cardiology. ${ }^{14}$ The luminal/intimal borders were contoured manually to determine the lumen CSA. The EEM CSA, representing the area encompassed by the medial-adventitial border, was measured by tracing the leading edge of the adventitia to determine the CSA of the vessel. Conventional IVUS and integrated backscatter (IB)-IVUS measurements at 1-mm intervals were performed. The IB-IVUS technology has been described in detail in several previous reports. ${ }^{15-17}$ Data were captured on Visiwave console software and the tissue composition of coronary plaque was digitally labelled as lipid, fibrosis (fibrosis plus dense fibrosis), and calcification, according to the radiofrequency ultrasound backscatter signals. We previously reported good intraobserver and interobserver agreement regarding measurement of coronary plaque components. ${ }^{18}$ Changes in lipid profile, CRP, and grayscale and various IB-IVUS variables were calculated (follow-up value minus baseline value). Furthermore, EEM volume and lumen volume were calculated using Simpson's rule. PV represented EEM volume minus lumen volume. The volume of each plaque component was also calculated using Simpson's rule.

\section{Calculation of Endpoints}

The primary endpoint was the percent change in coronary PV and in lipid PV during the follow-up period. \%Change in $\mathrm{PV}$ was calculated as $\left[\left(\mathrm{PV}_{\text {follow-up }}-\mathrm{PV}\right.\right.$ baseline $) / \mathrm{PV}$ baseline $] \times$ 100 , where $\mathrm{PV}=\Sigma($ EEM CSA-lumen CSA $)$. Lipid PV was calculated as $\Sigma$ lipid plaque CSA. The secondary endpoints were absolute change in $\% \mathrm{PV}$ and in normalized PV (NPV). \%PV was calculated using the following formula: $\% \mathrm{PV}=[\mathrm{PV} / \Sigma($ EEM CSA $)] \times 100$. NPV was calculated as $\mathrm{PV} \times[$ LMED/LMEASURED], where LMED=the median observed length in all subjects and LMEASURED=the observed length for each plaque. The intra- and inter-observer intra-class 


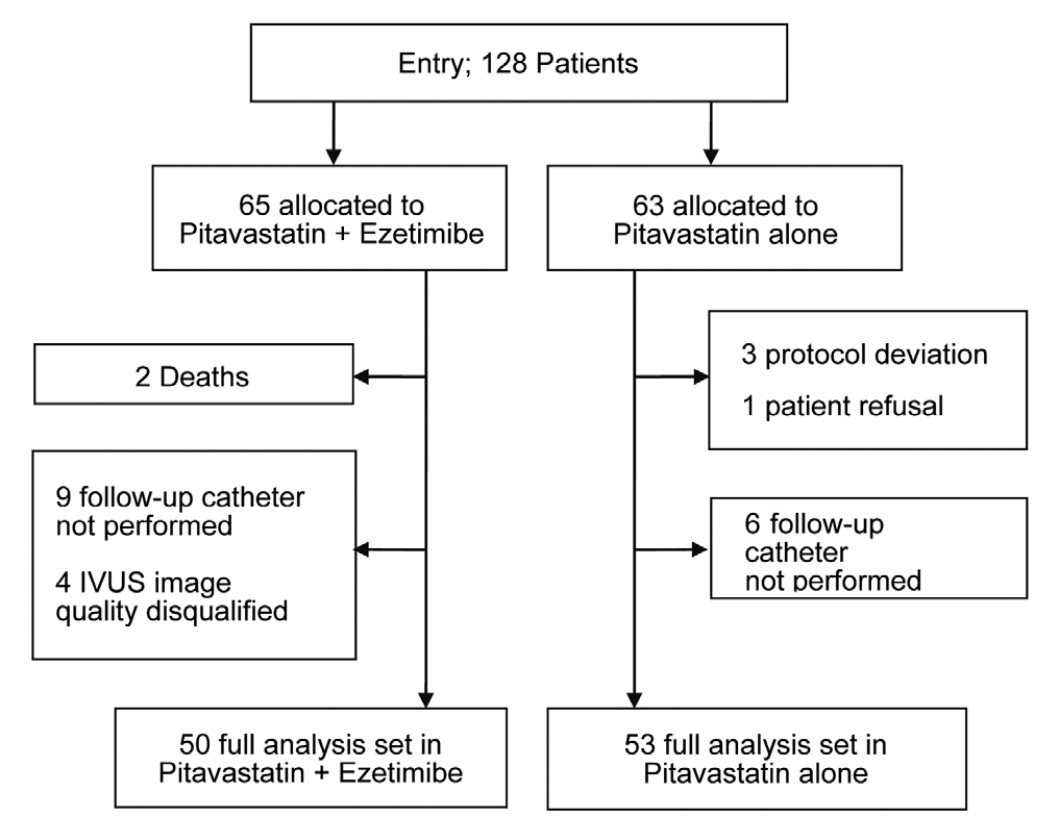

Figure 1. Study flowchart. IVUS, intravascular ultrasound.

correlation coefficients (ICC) for the vessel, lumen, and plaque areas were 0.999 and $0.999,0.996$ and 0.993 , and 0.993 and 0.991 , respectively, as reported previously. ${ }^{\mathbf{1 8}}$

\section{Definition of Major Adverse Cardiac Events (MACE)}

MACE were defined as a composite of cardiac death, myocardial infarction, or any repeat revascularization during the study period.

\section{Statistical Analysis}

Quantitative data are expressed as mean \pm SD for continuous variables and frequencies for categorical variables. The Kolmogorov-Smirnov test was used to determine the pattern of data distribution (normal or skewed). For normally distributed data, differences between patient groups were tested using Student's t-test. Given that Student's t-test assumes homogeneity of variance, Levene's test was applied to assess the equality of variances in each group. For Leven test $\mathrm{P}<0.05$, Welch's t-test was used. For skewed data distribution, between-group differences were tested using MannWhitney U-test. Categorical data were compared using the chi-squared test or Fisher's exact test as appropriate.

For difference in percent change in $\mathrm{PV}$, a sample size $\geq 43$ patients in each treatment group was required to provide $80 \%$ power at a 2 -sided $\alpha$ of 0.05 to demonstrate a relative $5.7 \%$ difference, ${ }^{19}$ assuming a $9.3 \%$ standard deviation. ${ }^{20}$ To allow for $20 \%$ dropout rate, we recruited 120 patients.

All tests were 2-tailed, and $\mathrm{P}<5 \%$ was considered to reflect statistical significance. Statistical analysis was performed using the Statistical Package for Social Sciences (IBM SPSS statistics 22.0 for Windows, SPSS, Chicago, IL, USA).

\section{Results}

\section{Patient Characteristics}

Figure 1 shows the number of patients at each step of the selection and randomization process and the reasons for discontinuation. Between October 2010 and September 2012, 128 patients at 10 centers were randomized and received the study drug, and $103(80.5 \%)$ had evaluable IVUS data at both baseline and follow-up. Thus, the full analysis set (FAS) consisted of 50 patients in the ezetimibe plus statin (combination) group and 53 patients in the statin monotherapy group (Table 1). The mean time interval between baseline and follow-up was $10.0 \pm 1.9$ months The 2 treatment groups were well balanced with regard to baseline demographics and clinical characteristics. Mean age was $63.2 \pm 10.8$ years, $79 \%$ of patients were men, $30 \%$ had diabetes, $72 \%$ had STEMI, and drug-eluting stents were used in $28 \%$ and bare-metal stents in $69 \%$. According to the study protocol, all patients received statin treatment. There were no significant differences between the groups with regard to medication at discharge.

\section{Effects of Lipid-Lowering Therapy}

Table 2 summarizes the laboratory results for the FAS group conducted at baseline and 10-month follow-up. Mean LDL-C decreased significantly from 123 to $64 \mathrm{mg} / \mathrm{dL}$ in the combination group $(\mathrm{n}=50)$ and from 126 to $87 \mathrm{mg} / \mathrm{dL}$ in the statin alone group $(\mathrm{n}=53$; between-group difference, $16.9 \%, \mathrm{P}<0.0001)$. Thus, the combination therapy produced greater reduction in LDL-C compared with statin alone $(-45.7 \%$ vs. $-28.8 \%, \mathrm{P}<0.0001)$. Furthermore, highsensitivity CRP (hs-CRP) decreased significantly from $1.75 \pm 2.16 \mathrm{mg} / \mathrm{dL}$ at baseline to $0.12 \pm 0.19 \mathrm{mg} / \mathrm{dL}$ after 10 -month therapy in the combination group $(\mathrm{P}<0.0001)$, and from $2.92 \pm 3.57 \mathrm{mg} / \mathrm{dL}$ to $0.14 \pm 0.22 \mathrm{mg} / \mathrm{dL}$ in the statin monotherapy $(\mathrm{P}<0.0001)$. Similarly, pentraxin 3 decreased significantly from baseline to 10-month follow-up in both groups. The reductions in hs-CRP and pentraxin 3, however, were similar between the 2 groups.

\section{Effects of Lipid-Lowering Therapy on IVUS Endpoints}

Changes in grayscale and IB-IVUS parameters are summarized in Table 3. The primary endpoint, percent change in 


\begin{tabular}{lccc}
\hline Table 1. Baseline Clinical Characteristics & $\begin{array}{c}\text { Pitavastatin plus } \\
\text { ezetimibe }\end{array}$ & $\begin{array}{c}\text { Pitavastatin } \\
\text { monotherapy }\end{array}$ & P-value \\
$\mathrm{n}$ & 50 & 53 & 0.941 \\
Age (years) & $63 \pm 10$ & $63 \pm 12$ & 0.559 \\
Male sex & $41(82)$ & $41(77)$ & 0.064 \\
Hypertension & $23(46)$ & $34(64)$ & 0.924 \\
Diabetes mellitus & $10(20)$ & $11(21)$ & 0.653 \\
Current smoking & $22(44)$ & $20(38)$ & 0.362 \\
STEMI & $38(76)$ & $36(68)$ & 0.305 \\
Family history of CAD & $9(18)$ & $14(26)$ & 0.977 \\
No. diseased vessels & & & \\
1 & $32(64)$ & $35(66)$ & \\
2 & $12(24)$ & $12(23)$ & \\
3 & $6(12)$ & $6(11)$ & 0.610 \\
Medication & & & 0.101 \\
Nitrates & $2(4)$ & $1(2)$ & 0.876 \\
Calcium channel blockers & $13(26)$ & $7(13)$ & 0.689 \\
$\beta$-blockers & $30(60)$ & $31(51)$ & 0.886 \\
AllRA & $14(28)$ & $13(25)$ & $30(57)$ \\
ACEl & $29(58)$ & $36 E$. & \\
\hline
\end{tabular}

Data given as mean \pm SD or $n(\%)$. AlIRA, angiotensin II receptor antagonist; $A C E I$, angiotensin-converting enzyme inhibitor; CAD, coronary artery disease; STEMI, ST-segment elevation myocardial infarction.

\begin{tabular}{|c|c|c|c|}
\hline & $\begin{array}{c}\text { Pitavastatin plus } \\
\text { ezetimibe }\end{array}$ & $\begin{array}{l}\text { Pitavastatin } \\
\text { monotherapy }\end{array}$ & P-value \\
\hline $\mathbf{n}$ & 50 & 53 & \\
\hline \multicolumn{4}{|l|}{ Lipid profile } \\
\hline \multicolumn{4}{|l|}{ Baseline } \\
\hline $\mathrm{TC}(\mathrm{mg} / \mathrm{dL})$ & $191 \pm 34$ & $196 \pm 37$ & 0.441 \\
\hline LDL-C (mg/dL) & $123 \pm 32$ & $126 \pm 33$ & 0.598 \\
\hline $\mathrm{HDL}-\mathrm{C}(\mathrm{mg} / \mathrm{dL})$ & $45 \pm 14$ & $46 \pm 11$ & 0.597 \\
\hline $\mathrm{TG}(\mathrm{mg} / \mathrm{dL})$ & $109 \pm 64$ & $112 \pm 52$ & 0.800 \\
\hline \multicolumn{4}{|l|}{ 10-month follow-up } \\
\hline TC (mg/dL) & $132 \pm 20^{\star \star \star}$ & $156 \pm 29^{\star \star \star}$ & $<0.0001$ \\
\hline LDL-C (mg/dL) & $64 \pm 18^{\star \star \star}$ & $87 \pm 21^{* \star *}$ & $<0.0001$ \\
\hline $\mathrm{HDL}-\mathrm{C}(\mathrm{mg} / \mathrm{dL})$ & $49 \pm 12^{*}$ & $49 \pm 15$ & 0.853 \\
\hline $\mathrm{TG}(\mathrm{mg} / \mathrm{dL})$ & $108 \pm 53$ & $129 \pm 77$ & 0.107 \\
\hline \multicolumn{4}{|c|}{ Change in lipid profiles } \\
\hline$\Delta \mathrm{TC}(\mathrm{mg} / \mathrm{dL})$ & $-59 \pm 33$ & $-41 \pm 34$ & 0.017 \\
\hline$\Delta \mathrm{LDL}-\mathrm{C}(\mathrm{mg} / \mathrm{dL})$ & $-58 \pm 27$ & $-40 \pm 31$ & 0.005 \\
\hline$\Delta \mathrm{HDL}-\mathrm{C}(\mathrm{mg} / \mathrm{dL})$ & $4 \pm 10$ & $2 \pm 12$ & 0.358 \\
\hline$\Delta \mathrm{TG}(\mathrm{mg} / \mathrm{dL})$ & $-3 \pm 71$ & $18 \pm 65$ & 0.152 \\
\hline \multicolumn{4}{|l|}{ Hs-CRP (mg/dL) } \\
\hline Baseline & $1.8 \pm 2.2$ & $2.9 \pm 3.6$ & 0.181 \\
\hline 10-month follow-up & $0.1 \pm 0.2^{\star \star \star}$ & $0.1 \pm 0.2^{* * *}$ & 0.715 \\
\hline Change & $-1.7 \pm 2.2$ & $-2.8 \pm 3.6$ & 0.298 \\
\hline \multicolumn{4}{|l|}{ Pentraxin 3 (ng/mL) } \\
\hline Baseline & $5.8 \pm 5.0$ & $6.1 \pm 4.3$ & 0.737 \\
\hline 10-month follow-up & $2.4 \pm 2.1^{\star \star \star}$ & $2.4 \pm 3.1^{* \star \star}$ & 0.992 \\
\hline Change & $-3.4 \pm 4.9$ & $-3.7 \pm 4.3$ & 0.795 \\
\hline Peak CK (IU/L) & $1,940 \pm 1,973$ & $1,916 \pm 2,318$ & 0.955 \\
\hline
\end{tabular}

Data given as $n$, mean $\pm S D$, or $n(\%) .{ }^{*} P<0.05,{ }^{* * *} \mathrm{P}<0.001$ (baseline vs. follow-up). $C K$, creatine kinase; HDL-C, high-density lipoprotein cholesterol; hs-CrP, high-sensitivity C-reactive protein; LDL-C, low-density lipoprotein cholesterol; TC, total cholesterol; TG, triglycerides. 


\begin{tabular}{|c|c|c|c|}
\hline & $\begin{array}{l}\text { Pitavastatin plus } \\
\text { ezetimibe }\end{array}$ & $\begin{array}{l}\text { Pitavastatin } \\
\text { monotherapy }\end{array}$ & P-value \\
\hline $\mathbf{n}$ & 50 & 53 & \\
\hline Non-culprit vessels analysis & $37(74)$ & $43(81)$ & 0.385 \\
\hline \multicolumn{4}{|l|}{ Baseline } \\
\hline Length of analyzed lesion (mm) & $38 \pm 19$ & $41 \pm 18$ & 0.396 \\
\hline EEM volume $\left(\mathrm{mm}^{3}\right)$ & $534 \pm 375$ & $585 \pm 335$ & 0.465 \\
\hline Lumen volume $\left(\mathrm{mm}^{3}\right)$ & $301 \pm 211$ & $334 \pm 210$ & 0.425 \\
\hline $\mathrm{PV}\left(\mathrm{mm}^{3}\right)$ & $233 \pm 175$ & $251 \pm 155$ & 0.580 \\
\hline Percent atheroma volume (\%) & $44.3 \pm 9.4$ & $43.9 \pm 10.6$ & 0.816 \\
\hline \multicolumn{4}{|l|}{ 8-12-month follow-up } \\
\hline Length of analyzed lesion (mm) & $38 \pm 19$ & $41 \pm 19$ & 0.374 \\
\hline EEM volume $\left(\mathrm{mm}^{3}\right)$ & $523 \pm 382^{*}$ & $574 \pm 335^{*}$ & 0.472 \\
\hline Lumen volume $\left(\mathrm{mm}^{3}\right)$ & $301 \pm 219$ & $334 \pm 211$ & 0.438 \\
\hline $\mathrm{PV}\left(\mathrm{mm}^{3}\right)$ & $222 \pm 175^{\star \star}$ & $240 \pm 153^{\star *}$ & 0.577 \\
\hline Percent PV (\%) & $42.9 \pm 9.6^{\star \star \star}$ & $42.0 \pm 10.0^{* *}$ & 0.650 \\
\hline \multicolumn{4}{|l|}{ Change } \\
\hline$\Delta$ EEM volume $\left(\mathrm{mm}^{3}\right)$ & $-11 \pm 39$ & $-12 \pm 35$ & 0.973 \\
\hline$\Delta$ Lumen volume $\left(\mathrm{mm}^{3}\right)$ & $0 \pm 29$ & $0 \pm 26$ & 0.966 \\
\hline$\Delta \mathrm{PV}\left(\mathrm{mm}^{3}\right)$ & $-11 \pm 22$ & $-11 \pm 23$ & 0.997 \\
\hline Percent change in PV (\%) & $-5.1 \pm 12.2$ & $-6.2 \pm 13.8$ & 0.664 \\
\hline \multicolumn{4}{|l|}{ Absolute change } \\
\hline Normalized PV $\left(\mathrm{mm}^{3}\right)$ & $-6.9 \pm 25.6$ & $-8.3 \pm 30.8$ & 0.798 \\
\hline Percent PV (\%) & $-1.5 \pm 4.3$ & $-1.9 \pm 4.7$ & 0.639 \\
\hline
\end{tabular}

Data given as $n$, mean $\pm S D$, or $n(\%)$. ${ }^{*} P<0.05$, ${ }^{*} \mathrm{P}<0.01,{ }^{* * *} \mathrm{P}<0.001$ (baseline vs. follow-up). EEM, external elastic membrane; IVUS, intravascular ultrasound; PV, plaque volume.

\begin{tabular}{|c|c|c|c|}
\hline & $\begin{array}{c}\text { Pitavastatin plus } \\
\text { ezetimibe }\end{array}$ & $\begin{array}{l}\text { Pitavastatin } \\
\text { monotherapy }\end{array}$ & P-value \\
\hline $\mathbf{n}$ & 50 & 53 & \\
\hline \multicolumn{4}{|l|}{ Baseline } \\
\hline Lipid PV $\left(\mathrm{mm}^{3}\right)$ & $133 \pm 132$ & $140 \pm 106$ & 0.750 \\
\hline Fibrous PV (mm³) & $98 \pm 61$ & $108 \pm 65$ & 0.415 \\
\hline Calcium volume $\left(\mathrm{mm}^{3}\right)$ & $2.4 \pm 1.7$ & $2.8 \pm 2.7$ & 0.368 \\
\hline \multicolumn{4}{|l|}{ Follow-up at 10 months } \\
\hline Lipid PV $\left(\mathrm{mm}^{3}\right)$ & $125 \pm 113$ & $127 \pm 101$ & 0.927 \\
\hline Fibrous PV $\left(\mathrm{mm}^{3}\right)$ & $94 \pm 77$ & $110 \pm 69$ & 0.279 \\
\hline Calcium volume $\left(\mathrm{mm}^{3}\right)$ & $2.4 \pm 2.0$ & $2.9 \pm 2.6$ & 0.237 \\
\hline \multicolumn{4}{|l|}{ Percent change } \\
\hline Lipid PV (\%) & $4.3 \pm 39.1$ & $-3.0 \pm 43.3$ & 0.374 \\
\hline Fibrous PV (\%) & $-1.0 \pm 44.8$ & $9.1 \pm 50.2$ & 0.286 \\
\hline Calcification volume (\%) & $66.9 \pm 259.9$ & $148.3 \pm 351.7$ & 0.187 \\
\hline
\end{tabular}

Data given as mean \pm SD. IB-IVUS, integrated backscatter intravascular ultrasound; PV, plaque volume.

$\mathrm{PV}$, was significantly lower in both groups (combination group, $-5.1 \%$; statin group, $-6.2 \%, \mathrm{P}<0.01$, each), but the between-group difference was not significant $(\mathrm{P}=0.66$; Table 3). Similarly, the percent change in lipid PV was not significantly different between the 2 groups ( 4.3 vs. $-3.0 \%$, $\mathrm{P}=0.37$, Table 4). The secondary endpoint, absolute change in percent atheroma volume, was not different between the 2 groups $(-1.5$ vs. $-1.9 \%, \mathrm{P}=0.64)$.

\section{Subgroup Analysis}

We observed no significant difference in the primary endpoint with regard to age, sex, coronary risk factors, or angiographic or IVUS variables (Table 5).

Lipid Profile and Percent Change in Plaque Volume There were no significant correlations between percent change in LDL-C during the study period and percent change in PV in the combination group or the statin monotherapy group (Figure 2A). Similarly, no significant correlations were observed between percent change in LDL-C and percent change in lipid PV in either group (Figure 2B).

Markers of Cholesterol Synthesis and Absorption: IVUS Endpoints In a subgroup of patients $(n=77)$, serum concentration of the markers of cholesterol synthesis (lathosterol) and absorption (campesterol and sitosterol) was measured. 


\begin{tabular}{|c|c|c|c|}
\hline & \multicolumn{2}{|c|}{ \%change in coronary PV } & \multirow[b]{2}{*}{ P-value } \\
\hline & $\begin{array}{c}\text { Pitavastatin plus } \\
\text { ezetimibe }\end{array}$ & $\begin{array}{l}\text { Pitavastatin } \\
\text { monotherapy }\end{array}$ & \\
\hline All $(n=103)$ & $-5.1 \pm 12.2$ & $-6.2 \pm 13.8$ & 0.664 \\
\hline \multicolumn{4}{|l|}{ Lesion length } \\
\hline$\geq 10 \mathrm{~mm}(\mathrm{n}=91)$ & $-6.2 \pm 10.1$ & $-4.3 \pm 9.2$ & 0.385 \\
\hline$\geq 30 \mathrm{~mm}(\mathrm{n}=72)$ & $-4.3 \pm 10.3$ & $-4.9 \pm 9.1$ & 0.809 \\
\hline \multicolumn{4}{|l|}{ Age } \\
\hline$<70$ years $(n=72)$ & $-5.5 \pm 13.0$ & $-6.7 \pm 16.0$ & 0.717 \\
\hline$\geq 70$ years $(n=32)$ & $-4.3 \pm 9.4$ & $-5.3 \pm 9.1$ & 0.764 \\
\hline \multicolumn{4}{|l|}{ Sex } \\
\hline Male $(n=82)$ & $-3.8 \pm 12.6$ & $-6.0 \pm 15.5$ & 0.472 \\
\hline Female $(n=21)$ & $-11.0 \pm 7.9$ & $-6.7 \pm 6.1$ & 0.182 \\
\hline \multicolumn{4}{|l|}{ Diagnosis } \\
\hline STEMI $(n=74)$ & $-5.9 \pm 11.0$ & $-6.0 \pm 15.9$ & 0.974 \\
\hline Non-STEACS $(\mathrm{n}=29)$ & $-2.5 \pm 15.7$ & $-6.7 \pm 8.4$ & 0.368 \\
\hline \multicolumn{4}{|l|}{ Current smoker } \\
\hline Yes $(n=43)$ & $-3.1 \pm 8.1$ & $-7.1 \pm 19.4$ & 0.387 \\
\hline No $(n=60)$ & $-6.6 \pm 14.6$ & $-5.6 \pm 8.79$ & 0.752 \\
\hline \multicolumn{4}{|l|}{ Hypertension } \\
\hline Yes $(n=57)$ & $-2.9 \pm 9.7$ & $-5.5 \pm 16.4$ & 0.497 \\
\hline No $(n=46)$ & $-6.9 \pm 13.9$ & $-7.4 \pm 7.4$ & 0.889 \\
\hline \multicolumn{4}{|l|}{ DM } \\
\hline Yes $(n=21)$ & $-6.4 \pm 8.0$ & $-13.0 \pm 24.7$ & 0.428 \\
\hline No $(n=82)$ & $-4.7 \pm 13.0$ & $-4.4 \pm 8.9$ & 0.892 \\
\hline \multicolumn{4}{|l|}{ Family history of CAD } \\
\hline Yes $(n=23)$ & $0.8 \pm 14.8$ & $-1.5 \pm 10.4$ & 0.669 \\
\hline No $(n=80)$ & $-6.3 \pm 11.3$ & $-7.9 \pm 14.6$ & 0.603 \\
\hline \multicolumn{4}{|l|}{ Analyzed vessel } \\
\hline Culprit vessel $(n=23)$ & $-2.3 \pm 5.2$ & $-0.6 \pm 4.1$ & 0.616 \\
\hline Non-culprit vessel $(n=80)$ & $-5.0 \pm 10.5$ & $-7.1 \pm 14.4$ & 0.458 \\
\hline \multicolumn{4}{|l|}{ Analyzed location } \\
\hline $\operatorname{LAD}(n=56)$ & $-3.0 \pm 13.7$ & $-6.0 \pm 8.4$ & 0.347 \\
\hline LCX/RCA $(n=47)$ & $-8.0 \pm 8.9$ & $-6.3 \pm 17.8$ & 0.700 \\
\hline \multicolumn{4}{|l|}{ No. diseased vessels } \\
\hline 1 vessel $(n=67)$ & $-5.5 \pm 12.7$ & $-6.4 \pm 8.7$ & 0.734 \\
\hline$\geq 2$ vessels $(n=36)$ & $-4.2 \pm 11.6$ & $-5.7 \pm 20.9$ & 0.794 \\
\hline \multicolumn{4}{|l|}{ Analyzed plaque vulnerability } \\
\hline With lipid pool $(n=37)$ & $5.8 \pm 11.0$ & $-3.0 \pm 8.3$ & 0.399 \\
\hline Without lipid pool $(n=65)$ & $-3.4 \pm 11.5$ & $-7.7 \pm 15.7$ & 0.223 \\
\hline
\end{tabular}

Data given as mean $\pm S D$. CAD, coronary artery disease; $D M$, diabetes mellitus; $L A D$, left anterior descending coronary artery; LCX, left circumflex coronary artery; non-STEACS, non-ST-elevation acute coronary syndrome; PV, plaque volume; RCA, right coronary artery; STEMI, ST-segment elevation acute myocardial infarction.

Patients were stratified according to the medians of lathosterol, campesterol, and sitosterol (Table 6). In any subgroup, the primary and secondary endpoints were not significantly different between the combination group and the statin alone group on quantitative grayscale IVUS.

MACE The treated group consisted of all patients who received any dose of study medication (128 randomized patients) and was considered for analysis of safety and adverse events. As expected, given the relatively small number of patients, the number of patients who developed MACE during the 10-month follow-up was not different between the 2 groups ( 9 patients in the combination group and 6 in the statin monotherapy group, $\mathrm{P}=0.63$ ). There were 2 deaths (cardiac death in 1 and non-cardiac in another) in the combination group vs. none in the monotherapy group $(\mathrm{P}=0.50)$. Repeat revascularization was performed in $10.7 \%(7 / 65)$ of the combination group and in $9.5 \%(6 / 63)$ of the statin alone group $(\mathrm{P}=0.82)$.

\section{Discussion}

The main findings of this study are as follows: (1) the combination of ezetimibe and statin reduced the level of LCL-C by $17 \%$; (2) coronary plaque regression was observed after 10-month treatment with either type of therapy (i.e., combination therapy or statin monotherapy); (3) the larger reduction in LDL-C with the combination therapy did not translate into greater regression of the 
A
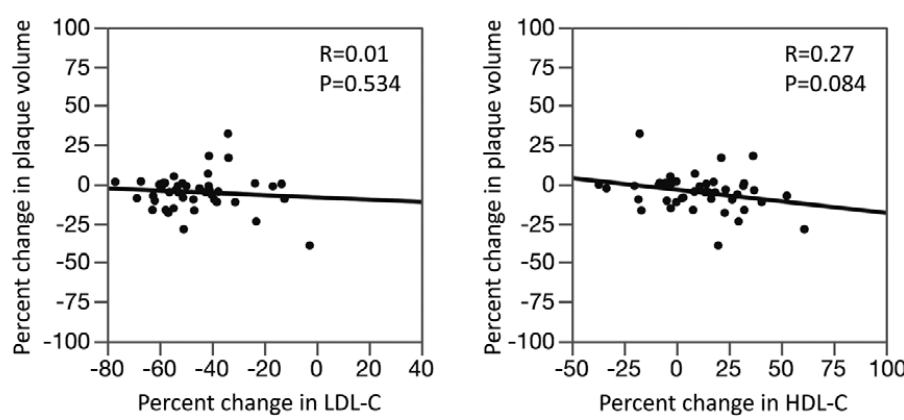

Pitavastatin Monotherapy
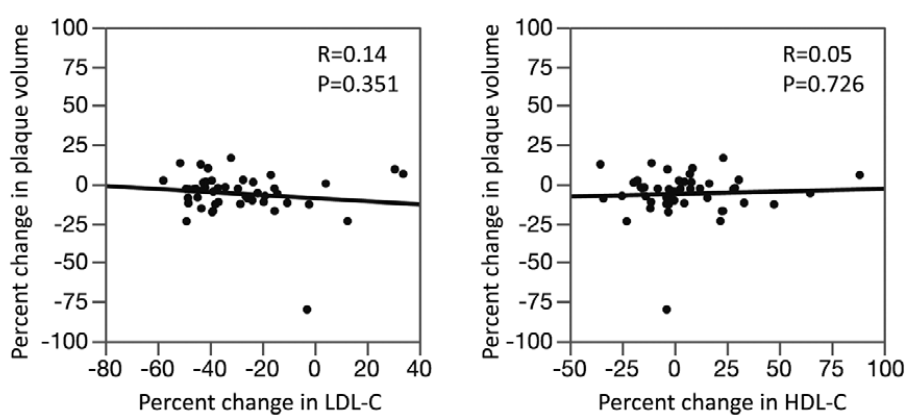
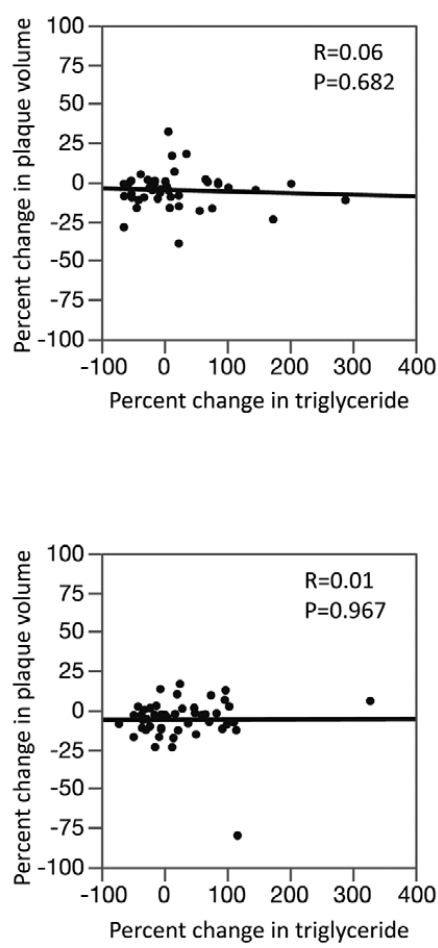

B

Pitavastatin plus Ezetimibe

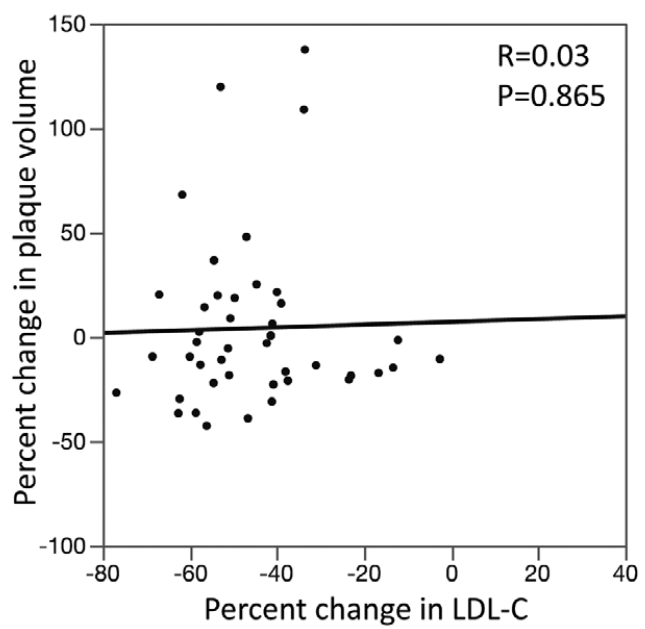

Pitavastatin Monotherapy

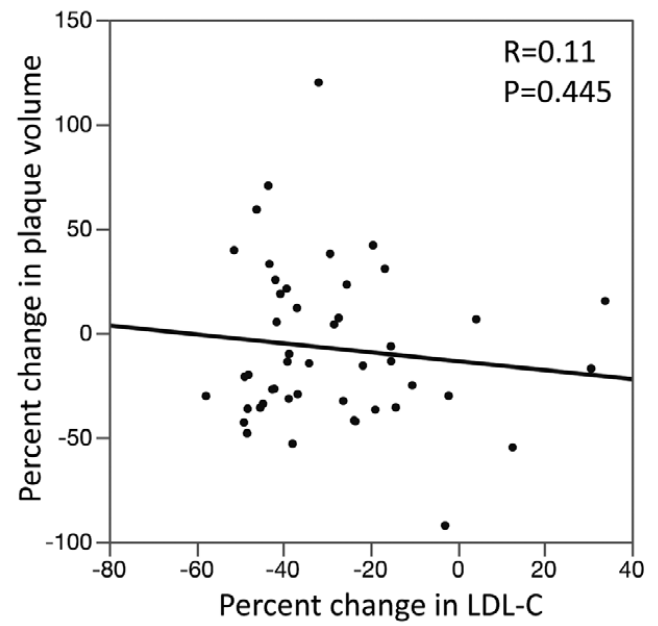

Figure 2. (A) Percent change in plaque volume vs. percent change in lipid profile and (B) percent change in low-density lipoprotein cholesterol (LDL-C) vs. percent change in plaque volume for the pitavastatin plus ezetimibe and pitavastatin monotherapy groups. HDL-C, high-density lipoprotein cholesterol.

non-culprit atherosclerotic plaque or change in plaque tissue characteristics in statin-naïve patients with ACS; and (4) the results were consistent across subgroups stratified according to the markers of cholesterol synthesis and absorption.
Theoretically, specific strategies targeting LDL-C in ACS may be beneficial with regard to the regression of atherosclerosis. ${ }^{21}$ Ezetimibe has been shown to reduce the levels of inflammatory markers, ${ }^{12,22}$ improve endothelial function, ${ }^{23}$ and lower LCL-C by approximately $15 \%$ beyond 


\begin{tabular}{|c|c|c|c|}
\hline & $\begin{array}{l}\text { Pitavastatin plus } \\
\text { ezetimibe }\end{array}$ & $\begin{array}{l}\text { Pitavastatin } \\
\text { monotherapy }\end{array}$ & P-value \\
\hline \multicolumn{4}{|l|}{ Sitosterol $<2.0 \mu \mathrm{g} / \mathrm{mL}(\mathrm{n}=32)^{\dagger}$} \\
\hline Percent change in PV (\%) & $-9.3 \pm 11.0$ & $-5.4 \pm 9.6$ & 0.296 \\
\hline Absolute change in normalized PV $\left(\mathrm{mm}^{3}\right)$ & $-15.5 \pm 32.3$ & $-0.3 \pm 24.7$ & 0.139 \\
\hline Absolute change in percent PV (\%) & $-2.6 \pm 4.5$ & $-1.6 \pm 2.8$ & 0.468 \\
\hline \multicolumn{4}{|l|}{ Sitosterol $\geq 2.0 \mu \mathrm{g} / \mathrm{mL}(\mathrm{n}=45)$} \\
\hline Percent change in PV (\%) & $-5.1 \pm 12.0$ & $-8.6 \pm 17.9$ & 0.457 \\
\hline Absolute change in normalized PV $\left(\mathrm{mm}^{3}\right)$ & $-5.3 \pm 21.3$ & $-15.8 \pm 38.1$ & 0.278 \\
\hline Absolute change in percent PV (\%) & $-1.5 \pm 4.3$ & $-1.9 \pm 4.7$ & 0.639 \\
\hline \multicolumn{4}{|l|}{ Campesterol $<4.3 \mu \mathrm{g} / \mathrm{mL}(\mathrm{n}=35)^{\dagger}$} \\
\hline Percent change in PV (\%) & $-8.4 \pm 11.1$ & $-5.9 \pm 7.9$ & 0.510 \\
\hline Absolute change in normalized $\mathrm{PV}\left(\mathrm{mm}^{3}\right)$ & $-13.5 \pm 30.5$ & $-3.9 \pm 14.7$ & 0.309 \\
\hline Absolute change in percent PV (\%) & $-2.5 \pm 4.6$ & $-1.5 \pm 3.0$ & 0.529 \\
\hline \multicolumn{4}{|l|}{ Campesterol $\geq 4.3 \mu \mathrm{g} / \mathrm{mL}(\mathrm{n}=42)$} \\
\hline Percent change in PV (\%) & $-5.1 \pm 12.3$ & $-8.1 \pm 17.8$ & 0.572 \\
\hline Absolute change in normalized $\mathrm{PV}\left(\mathrm{mm}^{3}\right)$ & $-5.0 \pm 21.2$ & $-12.8 \pm 40.3$ & 0.492 \\
\hline Absolute change in percent PV (\%) & $-0.53 \pm 4.3$ & $-2.8 \pm 5.8$ & 0.196 \\
\hline \multicolumn{4}{|l|}{ Lathosterol $<2.3 \mu \mathrm{g} / \mathrm{mL}(\mathrm{n}=38)^{\dagger}$} \\
\hline Percent change in PV (\%) & $-5.4 \pm 11.5$ & $-6.6 \pm 9.6$ & 0.727 \\
\hline Absolute change in normalized $\mathrm{PV}\left(\mathrm{mm}^{3}\right)$ & $-6.6 \pm 22.2$ & $-9.2 \pm 24.4$ & 0.729 \\
\hline Absolute change in percent PV (\%) & $-1.6 \pm 2.9$ & $-2.2 \pm 2.9$ & 0.473 \\
\hline \multicolumn{4}{|l|}{ Lathosterol $\geq 2.3 \mu \mathrm{g} / \mathrm{mL}(\mathrm{n}=39)$} \\
\hline Percent change in PV (\%) & $-8.7 \pm 11.7$ & $-8.2 \pm 19.6$ & 0.918 \\
\hline Absolute change in normalized $\mathrm{PV}\left(\mathrm{mm}^{3}\right)$ & $-13.7 \pm 31.6$ & $-10.8 \pm 42.6$ & 0.809 \\
\hline Absolute change in percent PV (\%) & $-1.9 \pm 5.8$ & $-2.5 \pm 6.6$ & 0.738 \\
\hline
\end{tabular}

Data given as mean \pm SD or ${ }^{t}$ median \pm SD. IVUS, intravascular ultrasound; PV, plaque volume.

the effects of concomitant statin therapy. ${ }^{12,24,25}$ The combination therapy, however, has also been reported to have no impact on the progression of carotid atherosclerosis in several other studies. For example, the Ezetimibe and Simvastatin in Hypercholesterolemia Enhances Atherosclerosis Regression (ENHANCE) clinical trial showed that the addition of ezetimibe to simvastatin did not reduce the intima-media thickness (IMT) of the carotid artery wall in patients with familial hypercholesterolemia, although a between-group difference in LDL-C $>15 \%$ was observed throughout the 24-month treatment period.12 Furthermore, the Arterial Biology for the Investigation of the Treatment Effects of Reducing Cholesterol 6-HDL and LDL Treatment Strategies (ARBITER 6-HALTS) trial compared the effect of 14-month treatment with niacin or ezetimibe on the carotid IMT in patients with coronary artery disease (CAD) or high risk for CAD. ${ }^{26}$ While niacin had greater efficacy with regard to the change in IMT compared with ezetimibe, ezetimibe led to paradoxical progression of IMT although it resulted in greater reduction in LDL-C with cumulative drug exposure, ${ }^{11}$ suggesting that ezetimibe may worsen arterial atherosclerosis. Although the effect of ezetimibe on coronary atherosclerosis may differ from that on carotid atherosclerosis, the present lack of anti-regression effect on coronary atherosclerosis by ezetimibe suggests that this cholesterol-lowering agent has different mechanisms of action compared with statins.

Lipid-lowering treatment is a first-line therapy for ACS, based on the findings of previous large-scale randomized trials of reductions in high LDL-C after high-dose statin therapy and reduced rate of atherosclerotic cardiovascular disease (ASCVD). The 2013 American Heart Association and American College of Cardiology (AHA/ACC) guidelines recommended high-intensity statin therapy as a secondary prevention strategy in patients with ASCVD, regardless of the LDL-C level. ${ }^{27}$ In the present study, no correlation was observed between LDL-C reduction and plaque regression. In IMPROVE-IT, the addition of ezetimibe to high-dose statin therapy resulted in a significantly lower risk of cardiovascular events, ${ }^{8}$ suggesting that lipidlowering therapy other than statin may have anti-atherosclerotic effect. The PRECISE-IVUS trial was the first to show reduction of coronary atherosclerosis with dual lipidlowering therapy of ezetimibe and statin compared with statin alone. ${ }^{9}$ In contrast, the present results do not support the anti-atherosclerotic effect of ezetimibe when added to statin in statin-naïve patients with ACS. There are 3 main differences between the PRECISE-IVUS trial and the present study: first, they increased atorvastatin by titration with a treatment goal of LDL-C $<70 \mathrm{mg} / \mathrm{dL}$, while we selected a fixed dose of pitavastatin $2 \mathrm{mg} /$ day. As a result, the present achieved LDL-C level in the ezetimibe-statin combination group $(64 \pm 18$ vs. $63 \pm 16 \mathrm{mg} / \mathrm{dL})$ was similar to that in the PRECISE-IVUS trial, while that in the statin monotherapy group was higher $(87 \pm 21$ vs. $73 \pm 20 \mathrm{mg} / \mathrm{dL})$. Second, the PRECISE-IVUS trial enrolled a 2-fold larger number of patients and assessed non-culprit plaque in the culprit vessel for PCI, while we measured a 4-fold longer lesion length by examining non-culprit plaque in mainly "non-culprit vessels". And third, the PRECISE-IVUS trial enrolled both ACS and stable angina pectoris cohorts irrespective of previous statin use, whereas we enrolled 
patients with ACS and excluded those who had previously received statin treatment. It is therefore reasonable to conclude that the effects of the ezetimibe-statin combination therapy can vary according to the patient sample, ${ }^{28}$ method of treatment, and time of assessment of outcome. In a substudy of the PRECISE-IVUS trial, the IVUS endpoints were compared according to the presence or absence of statin pretreatment. ${ }^{29}$ In patients who received statin pretreatment, atorvastatin/ezetimibe combination had greater effect in reducing $\% \mathrm{PV}$ compared with atorvastatin alone $(-1.8 \%$ vs. $-0.1 \%, \mathrm{P}=0.002)$, whereas a non-significant effect was observed in patients who did not receive statin pretreatment $(-1.3$ vs. $-0.9, \mathrm{P}=0.12)$. In addition, West et al found that statin-naïve patients randomized to statin plus ezetimibe or statin alone had similar changes in peripheral PV despite a significant reduction in LDL-C. ${ }^{30}$ Furthermore, in the placebo arm of the Global Assessment of Plaque Regression With a PCSK9 Antibody as Measured by Intravascular Ultrasound (GLAGOV) trial, $98.3 \%$ of patients had statin pre-treatment, and baseline mean LDL-C was $92.4 \mathrm{mg} / \mathrm{dL}{ }^{31}$ Neither \%PV nor total PV changed from baseline to 78-week follow-up, indicating that the effect of statin on PV is not long-lasting. Thus, it is plausible that ezetimibe may exert a regressive effect when statin has lost its effect on PV. Alternatively, given that statin is known to increase LCL-C absorption, ${ }^{32}$ ezetimibe may exhibit its favorable effect on coronary atherosclerosis after a certain period of statin pretreatment. Given that we excluded patients with statin pretreatment, the true effect of ezetimibe on coronary atherosclerosis may have been altered or delayed in the present analysis. The present results question the value of simultaneous use of statin and ezetimibe in patients with ACS who did not receive statin at presentation.

\section{Study Limitations}

Several limitations must be given consideration. First, both baseline and follow-up IVUS data were available for only 103 of the 128 randomized patients $(80.5 \%)$. Patients who did not complete the trial may have different plaque behavior, although the acquisition rate in the present study is similar to that reported in previous trials., ${ }^{9,33,34}$ Second, the sample size of 103 patients was relatively small. Given the negative overall result, we cannot definitively rule out type II error. The percent reduction in PV was numerically smaller in the combination therapy group and it seems unlikely to be clinically meaningful, even with a larger number of patients. The present sample size, however, obviously lacks statistical power to prove non-inferiority and should be considered as hypothesis generating. Third, the period of 10 months may not have been long enough to detect differences in plaque behavior between the 2 groups. Nevertheless, the sample size was sufficient to detect significant plaque regression from baseline to 10-month follow-up in both groups. Fourth, we strongly encouraged the use of IVUS in the non-culprit vessel because it enables longer segment analysis, thereby increasing the reliability of the data. Given the safety issues, however, non-culprit plaques in culprit vessels were analyzed in 23 patients $(22 \%)$.

\section{Conclusions}

In statin-naïve patients with ACS, the combination of ezetimibe-statin therapy had no add-on effect on regression of coronary atherosclerosis compared with statin alone. A larger number of patients including a heterogeneous population with and without statin pretreatment and long-term follow-up is needed to establish the true effects of such therapy on coronary atherosclerosis.

\section{Acknowledgments}

This work was supported in part by a grant from Japan Heart Foundation (12090006). The funding agency had no role in the design or conduct of the study, in the collection, analysis, or interpretation of the data, or in the preparation, review, or approval of the manuscript.

\section{Disclosures}

K.H. has received remuneration for lectures from Daiichi-Sankyo, Terumo, Amgen Astellas BioPharma, Sanofi, and Boston Scientific Japan; and research grant from Daiichi-Sankyo, MSD, Terumo, Teijin Parma, Nipro, and St. Jude Medical Japan. T. Murohara has received remuneration for lectures from Bayer, Daiichi-Sankyo, Dainippon-Sumitomo, Kowa, MSD, Mitsubishi-Tanabe, Nihon Boehringer Ingelheim, Novartis, Pfizer, Sanofi-aventis, and Takeda; and research grant from Astellas, Daiichi-Sankyo, DainipponSumitomo, Kowa, MSD, Mitsubishi-Tanabe, Nippon Boehringer Ingelheim, Novartis, Otsuka, Pfizer Japan, Sanofi-aventis, Takeda, and Teijin. H.I. has received remuneration for lectures from Astellas, AstraZeneca, and Daiichi-Sankyo. K. Kimura has received remuneration for lectures from AstraZeneca, Toa Eiyo, MSD, Bayer and Daiichi-Sankyo; and research grant from Toa Eiyo, Bayer, MSD, Astellas, AstraZeneca, Sanofi, Eli Lilly Japan, Research Institute for Production Development, Pfizer, Shionogi, Kowa, Daiichi-Sankyo, Mitsubishi-Tanabe, Nihon Boehringer-Ingelheim, Takeda, Otsuka, and Ono. The other authors declare no conflict of interest.

\section{References}

1. LaRosa JC, Grundy SM, Waters DD, Shear C, Barter P, Fruchart JC, et al. Intensive lipid lowering with atorvastatin in patients with stable coronary disease. $N$ Engl J Med 2005; 352: $1425-1435$.

2. Cannon CP, Braunwald E, McCabe CH, Rader DJ, Rouleau JL, Belder R, et al. Intensive versus moderate lipid lowering with statins after acute coronary syndromes. $N$ Engl J Med 2004; 350: $1495-1504$.

3. Schwartz GG, Olsson AG, Ezekowitz MD, Ganz P, Oliver MF, Waters $\mathrm{D}$, et al. Effects of atorvastatin on early recurrent ischemic events in acute coronary syndromes: The MIRACL study: A randomized controlled trial. JAMA 2001; 285: 1711-1718.

4. Cholesterol Treatment Trialists' (CTT) Collaboration, Fulcher J, O'Connell R, Voysey M, Emberson J, Blackwell L, Mihaylova $\mathrm{B}$, et al. Efficacy and safety of LDL-lowering therapy among men and women: Meta-analysis of individual data from 174,000 participants in 27 randomised trials. Lancet 2015; 385: $1397-$ 1405.

5. McKenney JM. Optimizing LDL-C lowering with statins. Am J Ther 2004; 11: 54-59.

6. Okada K, Iwahashi N, Endo T, Himeno H, Fukui K, Kobayashi $\mathrm{S}$, et al. Long-term effects of ezetimibe-plus-statin therapy on low-density lipoprotein cholesterol levels as compared with double-dose statin therapy in patients with coronary artery disease. Atherosclerosis 2012; 224: 454-456.

7. Armitage J. The safety of statins in clinical practice. Lancet 2007; 370: $1781-1790$.

8. Cannon CP, Blazing MA, Giugliano RP, McCagg A, White JA, Theroux $\mathrm{P}$, et al. Ezetimibe added to statin therapy after acute coronary syndromes. N Engl J Med 2015; 372: 2387-2397.

9. Tsujita K, Sugiyama S, Sumida H, Shimomura H, Yamashita T, Yamanaga K, et al. Impact of dual lipid-lowering strategy with ezetimibe and atorvastatin on coronary plaque regression in patients with percutaneous coronary intervention: The multicenter randomized controlled PRECISE-IVUS Trial. J Am Coll Cardiol 2015; 66: 495-507.

10. Fleg JL, Mete M, Howard BV, Umans JG, Roman MJ, Ratner $\mathrm{RE}$, et al. Effect of statins alone versus statins plus ezetimibe on carotid atherosclerosis in type 2 diabetes: The SANDS (Stop Atherosclerosis in Native Diabetics Study) trial. $J$ Am Coll Cardiol 2008; 52: 2198-2205.

11. Taylor AJ, Villines TC, Stanek EJ. Paradoxical progression of atherosclerosis related to low-density lipoprotein reduction and exposure to ezetimibe. Eur Heart J 2012; 33: 2939-2945. 
12. Kastelein JJ, Akdim F, Stroes ES, Zwinderman AH, Bots ML, Stalenhoef AF, et al. Simvastatin with or without ezetimibe in familial hypercholesterolemia. N Engl J Med 2008; 358: 14311443.

13. Hibi K, Kimura T, Kimura K, Morimoto T, Hiro T, Miyauchi $\mathrm{K}$, et al. Clinically evident polyvascular disease and regression of coronary atherosclerosis after intensive statin therapy in patients with acute coronary syndrome: Serial intravascular ultrasound from the Japanese Assessment of Pitavastatin and Atorvastatin in Acute Coronary Syndrome (JAPAN-ACS) trial. Atherosclerosis 2011; 219: 743-749.

14. Mintz GS, Nissen SE, Anderson WD, Bailey SR, Erbel R, Fitzgerald PJ, et al. American College of Cardiology Clinical Expert Consensus Document on Standards for Acquisition, Measurement and Reporting of Intravascular Ultrasound Studies (IVUS): A report of the American College of Cardiology Task Force on Clinical Expert Consensus Documents. J Am Coll Cardiol 2001; 37: 1478-1492.

15. Kawasaki M, Takatsu H, Noda T, Sano K, Ito Y, Hayakawa K, et al. In vivo quantitative tissue characterization of human coronary arterial plaques by use of integrated backscatter intravascular ultrasound and comparison with angioscopic findings. Circulation 2002; 105: 2487-2492.

16. Okubo M, Kawasaki M, Ishihara Y, Takeyama U, Yasuda S, Kubota $\mathrm{T}$, et al. Tissue characterization of coronary plaques: Comparison of integrated backscatter intravascular ultrasound with virtual histology intravascular ultrasound. Circ J 2008; 72: $1631-1639$

17. Mitsuhashi T, Hibi K, Kosuge M, Morita S, Komura N, Kusama $\mathrm{I}$, et al. Relation between hyperinsulinemia and nonculprit plaque characteristics in nondiabetic patients with acute coronary syndromes. JACC Cardiovasc Imaging 2011; 4: 392-401.

18. Nakayama N, Hibi K, Endo M, Miyazawa A, Suzuki H, Maejima N, et al. Validity and reliability of new intravascular ultrasound analysis software for morphological measurement of coronary artery disease. Circ J 2013; 77: 424-431.

19. Okazaki S, Yokoyama T, Miyauchi K, Shimada K, Kurata T, Sato $\mathrm{H}$, et al. Early statin treatment in patients with acute coronary syndrome: Demonstration of the beneficial effect on atherosclerotic lesions by serial volumetric intravascular ultrasound analysis during half a year after coronary event: The ESTABLISH Study. Circulation 2004; 110: 1061 - 1068

20. Toi T, Taguchi I, Yoneda S, Kageyama M, Kikuchi A, Tokura $\mathrm{M}$, et al. Early effect of lipid-lowering therapy with pitavastatin on regression of coronary atherosclerotic plaque: Comparison with atorvastatin. Circ J 2009; 73: 1466-1472.

21. Matsushita K, Hibi K, Komura N, Akiyama E, Maejima N, Iwahashi $\mathrm{N}$, et al. Effects of 4 statins on regression of coronary plaque in acute coronary syndrome. Circ J 2016; 80: 1634-1643.

22. Pesaro AE, Serrano CV Jr, Fernandes JL, Cavalcanti AB, Campos AH, Martins HS, et al. Pleiotropic effects of ezetimibe/ simvastatin vs. high dose simvastatin. Int J Cardiol 2012; 158: $400-404$

23. Grigore L, Raselli S, Garlaschelli K, Redaelli L, Norata GD, Pirillo A, et al. Effect of treatment with pravastatin or ezetimibe on endothelial function in patients with moderate hypercholesterolemia. Eur J Clin Pharmacol 2013; 69: 341-346.

24. Robinson JG, Nedergaard BS, Rogers WJ, Fialkow J, Neutel JM, Ramstad D, et al. Effect of evolocumab or ezetimibe added to moderate- or high-intensity statin therapy on LDL-C lowering in patients with hypercholesterolemia: The LAPLACE-2 randomized clinical trial. JAMA 2014; 311: 1870-1882.

25. Ballantyne CM, Houri J, Notarbartolo A, Melani L, Lipka LJ, Suresh R, et al. Effect of ezetimibe coadministered with atorvastatin in 628 patients with primary hypercholesterolemia: A prospective, randomized, double-blind trial. Circulation 2003; 107: $2409-2415$.

26. Taylor AJ, Villines TC, Stanek EJ, Devine PJ, Griffen L, Miller $\mathrm{M}$, et al. Extended-release niacin or ezetimibe and carotid intimamedia thickness. $N$ Engl J Med 2009; 361: 2113-2122.

27. Stone NJ, Robinson JG, Lichtenstein AH, Bairey Merz CN, Blum CB, Eckel RH, et al. 2013 ACC/AHA guideline on the treatment of blood cholesterol to reduce atherosclerotic cardiovascular risk in adults: A report of the American College of Cardiology/American Heart Association Task Force on Practice Guidelines. Circulation 2014; 129: S1-S45.

28. Ueda Y, Hiro T, Hirayama A, Komatsu S, Matsuoka H, Takayama $\mathrm{T}$, et al. Effect of ezetimibe on stabilization and regression of intracoronary plaque: The ZIPANGU Study. Circ $J$ 2017; 81: $1611-1619$.

29. Tsujita K, Yamanaga K, Komura N, Sakamoto K, Sugiyama S, Sumida H, et al. Synergistic effect of ezetimibe addition on coronary atheroma regression in patients with prior statin therapy: Subanalysis of PRECISE-IVUS trial. Eur J Prev Cardiol 2016; 23: $1524-1528$.

30. West AM, Anderson JD, Meyer CH, Epstein FH, Wang H, Hagspiel KD, et al. The effect of ezetimibe on peripheral arterial atherosclerosis depends upon statin use at baseline. Atherosclerosis 2011; 218: $156-162$.

31. Nicholls SJ, Puri R, Anderson T, Ballantyne CM, Cho L, Kastelein JJ, et al. Effect of evolocumab on progression of coronary disease in statin-treated patients: The GLAGOV Randomized Clinical Trial. JAMA 2016; 316: 2373-2384

32. Okada K, Kimura K, Iwahashi N, Endo T, Himeno H, Fukui K, et al. Clinical usefulness of additional treatment with ezetimibe in patients with coronary artery disease on statin therapy: From the viewpoint of cholesterol metabolism. Circ J 2011; 75: $2496-$ 2504.

33. Hiro T, Kimura T, Morimoto T, Miyauchi K, Nakagawa Y, Yamagishi M, et al. Effect of intensive statin therapy on regression of coronary atherosclerosis in patients with acute coronary syndrome: A multicenter randomized trial evaluated by volumetric intravascular ultrasound using pitavastatin versus atorvastatin (JAPAN-ACS [Japan assessment of pitavastatin and atorvastatin in acute coronary syndrome] study). J Am Coll Cardiol 2009; 54: $293-302$.

34. Nicholls SJ, Ballantyne CM, Barter PJ, Chapman MJ, Erbel $\mathrm{RM}$, Libby $\mathrm{P}$, et al. Effect of two intensive statin regimens on progression of coronary disease. N Engl J Med 2011; 365: 2078 2087. 